\title{
Systematic Review of Energy-Efficient Scheduling Techniques in Cloud Computing
}

\author{
Shailesh S. Deore \\ Research Scholar \\ JJT University, Jhunjhunu \\ Rajasthan, India.
}

\author{
Ashok Narayan Patil, PhD. \\ Principal, Vasantrao Naik Arts and Science \\ College,Shahada \\ Maharashtra, India.
}

\begin{abstract}
A Cloud Computing is a promising paradigm for life of software and there survive. A lot of industries, companies and institutes decide to take benefits of their own cloud environments such as Infrastructure as a Service, Software as a Service, Platform as a Service which is heart of cloud computing. A Scheduler is required to schedule number of virtual machine as per virtual machine request from consumer. Scheduler schedules number of virtual machine request such that to save maximum energy and achieve greater degree of load balancing and less resource utilization. In this paper we review research work which is recently proposed by researchers on base of energy saving scheduling techniques which are recently developed.
\end{abstract}

\section{General Terms}

Cloud Computing, Scheduler

\section{Keywords}

Virtual machine request,Virtual machine, energy,Workload

\section{INTRODUCTION}

Now a days cloud computing is a promising computing paradigm which is to support virtualization, scalable resource utilization, para-virtulizaion and provide services such as Infrastructure as a Service, Software as a Service, Platform as a Service. Computer scientist predicting that cloud system is next generation operating system. Google engineers say a magnificent sentence maintains thousands of servers, warned that if power consumption continues to increase, power cost can easily overtake hardware cost by a large margin [2]. Consumer of cloud only needs internet connection. The burden of purchasing a new license copy, installing an application, update that copy monthly all burden can be removed, if users are cloud utilize [1],[6],[7]. Cloud can easily provide all these facilities. Cloud computing is open source for cloud developers [1]. The development of hypervisors Xen, KVM, VMware, Virtual box, Eucalyptus [3] etc. triggering development of commercial and Open source Cloud environment. It can offer services on base of energy, power Pay-per use model. In office environments, computers, Monitors account for highest energy consumption after lighting. Power dissipation is also a major concern in portable battery operated devices that have rapidly increased [2].

Green computing is a new inclination, trend for high end computer. For example, a 360 -Tflops supercomputer (such as IBM Blue Gene/L) with conventional processor requires 20MW to operate which is approximately equal to sum of 22,000 US households power consumption [15], [16], [14]. It is $0.5 \%$ of world total electricity usage [14], [17].

A variety of scheduling techniques have been developed in previous years. FCFS, Greedy[4], round robin[5], Priority
Scheduling, Shortest Job First, Back Filling in operating system for scheduling number of processes coming from users. All this algorithms are to save amount of energy. In this paper we focus survey of scheduling algorithms which are energy saving and maximum load distribution with less response time and minimum use of resource utilization.

\section{REVIEW OF RELATED WORK}

In this decade we refer many approaches viz. algorithm, methods, paradigms, techniques how to schedule virtual machines running on physical machines and also concentrate on energy consumption less, optimization , fully workload distribution , exploitation with physical machine ratability.

M. Devare et al [6] proposed a scheduling policy to implement Scheduler which assign number of virtual machine requests coming from consumer to virtual machines on the base of 'bully' and "non-bully" approach.

M.Sheikhalishahi et al [1] proposed multi-level and generalpurpose scheduling approach for energy efficient computing through software part of the green computing. The consolidation are well defined for IaaS cloud paradigm, however it is not limited to IaaS cloud model. The policies, models, algorithms and cloud pricing strategies are being discussed in general. The solutions in the context of Haizea are shown, through experiments. The big improvement in utilization and energy consumption is found as workloads are running with lower frequencies. The coincidence of energy consumption and utilization is improved.

M. Devare et al [7] discusses various hypervisors their development strategies and facilities for the Cloud systems. The Xen, VirtualBox, KVM and VMWare are being discussed. Moreover, it illustrates the comparison of cost reduction in electricity utilization due to virtualization and cloud systems.

Jiandun $\mathrm{Li}$ et al [8] introduce a hybrid energy-efficient scheduling algorithm for private clouds to reduce coming request response time, balance workload when data centre is running in low power mode and design algorithm on the base of pre power techniques and Least-load first algorithm.

Jiandun $\mathrm{Li}$ et al [9] introduce a hybrid energy-efficient scheduling algorithm for private clouds, concentrated on load balancing, Load migration on the base of state of virtual machines, count response time. If response time increases then energy also increases. So they minimised response time in their algorithm.

Saurabh Kumar Garg et al [10] presented a few scheduling approaches that could utilize heterogeneity across various data centres. Akshat Verma et al. [11] also present a dynamic power aware strategy. 
Chuliang Weng, Zhigang Wang et al [12] present hybrid scheduling framework for the CPU scheduling in the virtual machine monitor. Two types of applications are highthroughput type and concurrent type. Virtual machine sets concurrent type when majority of workload is concurrent applications in order to reduce cost of synchronization. Otherwise it is set to high-throughput type by default. Experiments and results show that framework and scheduling strategy is flexible to improve performance of virtual machine.

Gaurav Dhiman, Giacomo Marchetti et al [13] focus on vGreen developed MPC balance algorithm that concentrates on CPU and memory utilization decrease, amount of power save up to $15 \%$ to $20 \%$ of total power required. Power is directly propositional to energy so amount of energy also save.

Gregor Von Laszewski et al [14] proposed scheduling virtual machine in a compute cluster to reduce power consumption through Dynamic Voltage Frequency Scaling (DVFS), implementation of energy efficient algorithm to allocate virtual machine.

Bo Li, Jianxin Li et al [20] states Energy aware heuristic algorithm on base of distributes workload in virtual machine with minimum number of virtual machines or nodes required that workload. So that workload migration, workload resizes virtual machine migration these approaches are used in algorithm.

\section{METHODS}

\subsection{Hybrid Energy Efficient Algorithm}

Jiandun Li et al [8], [9], [22], [23], [24],[31] proposed hybrid energy efficient scheduling algorithm on pre-power technique and least-load first algorithm. Experiment and result show that approach can save maximum response time to conserve more energy and achieve higher load balancing. Virtual workflow is using Gaussian distribution assumption. Scheduling algorithm has following characteristics

- Intrusion not accepted.

- Workflow attributes regularly changes.

- Conserve energy, where as keeping response time is too long.

- Migration is applied.

Algorithm [9] is to reduce response time, to conserve energy and load balancing. If response time increases then energy also increase, so response time $(\mathrm{R})$ is directly propositional to energy $(\mathrm{E})$

$$
\mathrm{R} \propto \mathrm{T}
$$

This algorithm [9] contains three states of virtual machine busy, idle and sleep. Busy indicates virtual machine is running at high speed but uncompleted workload whereas idle indicates virtual machine running at high speed but no workload right now. And sleep is deep sleep. Sleep virtual machine requires more time to awake as compare to busy node. Algorithm calculates left capacity LC, least load LL, and unbalanced load UL. If LC < LL indicates left capacity running low, then start number of virtual machine at that instance, else if LC > UL shows least capacity running high perform migration. It searches minimum workload virtual machine called source virtual machine. If source virtual machine is in idle state then shutdown it. Then it finds new source virtual machine with minimum load and find target virtual machine with sub minimum load and then it applies migration , using migration maximum energy save. Figure.1 shows steps of hybrid energy efficient algorithm [9].

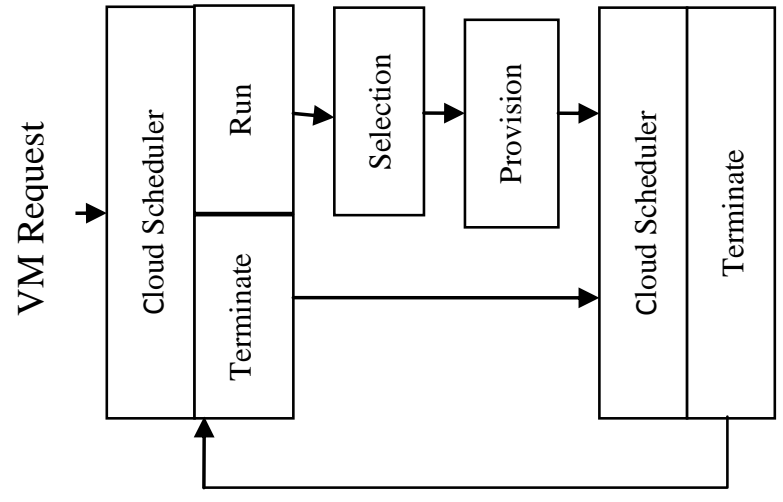

Fig 1. Procedure of hybrid energy-efficient scheduling algorithm

\subsection{Power-Aware Scheduling of Virtual Machines}

Gregor von Laszewski [14],[18],[19],[29],[30] state scheduling algorithm focus on scheduling virtual machines such that to calculate cluster to minimize power consumption via Dynamic Voltage frequency scaling (DVFS). Algorithm [14] is implemented in a simulator DVFS-enabled cluster by dynamically scaling supplied voltage. This is scenario operated for multiple voltages and frequencies, virtual machines are scheduled such that minimum voltage and frequency is required. Following parameters are considered operating point op, Energy E, power P, Voltage v, Frequency $\mathrm{s}$, processing element pe. Operating point $\mathrm{op}_{\mathrm{j}}$ is defined as follows [14],

$$
\mathrm{op}_{\mathrm{j}}=\left(\mathrm{v}^{\mathrm{op}}, \mathrm{s}^{\mathrm{op}}\right)
$$

Where,

$\mathrm{op}_{\mathrm{j}}$ is $\mathrm{j}^{\text {th }}$ operating point,

$\mathrm{v}^{\text {op }}$ voltage of $\mathrm{j}^{\text {th }}$ operating point

$\mathrm{s}^{\text {op }}$ is frequency of $\mathrm{j}^{\text {th }}$ operating point

$\mathrm{J}$ is total number of operating point

Therefore a set of operating point for a DVFS-enabled processor, op is defined as :

$$
\text { op }=U_{1<_{-}<_{-} J} \quad\left\{\mathrm{op}_{\mathrm{j}}\right\}
$$

Energy $E$ consumption of modern processor categorised into

$$
\mathrm{E}=\mathrm{E}_{\text {dynamic }}+\mathrm{E}_{\text {static }}
$$

Power consumption is defined $\mathrm{p}_{\text {dynamic }}$ calculate as

$$
\mathrm{P}_{\text {dynamic }}=\mathrm{ACv}^{2} \mathrm{~s}
$$

$\mathrm{A}=$ is percentage of active gates, $\mathrm{C}$ is total capacitance, $\mathrm{v}$ is supply voltage, $\mathrm{s}$ is processor frequency, time period $\nabla \mathrm{t}$ Then we defined

$$
\mathrm{E}_{\text {dynamic }}=\sum_{\mathrm{t}} \mathrm{P}_{\text {dynamic }} \cdot \Delta \mathrm{t}
$$

Whereas

$$
E_{\text {static }} \propto E_{\text {dynamic }}
$$

So whole power consumption is as follows

$$
\mathrm{E} \propto \mathrm{E}_{\text {dyanamic }}
$$

In conclusion, we have following performance model

$$
\mathrm{E} \propto \sum_{\mathrm{t}} \cdot \mathrm{v}^{2} . \Delta \mathrm{t}
$$

A cluster contains multiple compute nodes call as processing element pe at $\mathrm{k}^{\text {th }}$ cluster.

$$
\text { pek }=\left(o^{p e}, v^{\text {pe }}, s^{\text {pe }}\right)
$$


Virtual machine is defined as

$$
\mathrm{vm}_{\mathrm{i}}=\left(\mathrm{s}^{\mathrm{r}}, \Delta \mathrm{t}, \mathrm{t}^{\mathrm{r}}\right)
$$

Hence total virtual machine defined as

$$
\mathrm{VM}=\mathrm{U}_{1<\mathrm{i}<\mathrm{I}}\left\{\mathrm{vm}_{\mathrm{i}}\right\}
$$

Power consumption is directly proportional to supply voltage $\mathrm{f}:\left(\mathrm{vm}_{\mathrm{i}}\right) \rightarrow\left(\mathrm{pe}_{\mathrm{k}} \cdot \mathrm{sp}^{\mathrm{e}} \cdot \mathrm{pe}_{\mathrm{k}} \cdot \mathrm{sp}^{\mathrm{e}}\right), \mathrm{vm}_{\mathrm{i}} \in \mathrm{VM}, \mathrm{pe}_{\mathrm{k}} \in \mathrm{C}$

Where $E_{k}$ is the power consumption for $\mathrm{K}^{\mathrm{th}}$ processing element in a cluster.

Given a set of virtual machines VM and a cluster C defined above, find an optimal schedule $\mathrm{f}$, which minimize the power consumption cluster $\mathrm{C}$ :

$$
\mathrm{E}_{\min }=\min \sum_{\mathrm{k}=1}^{\mathrm{k}} \mathrm{E}_{\mathrm{k}}
$$

where $E_{k}$ is the power consumption of the $K_{t h}$ processing element in the cluster.

On the basis of above equations algorithm [14] is implemented. It Schedules virtual machines in a cluster minimizing power consumption. Supply voltage $\mathrm{v}$ of processor minimize by scaling down processor frequency $\mathrm{s}$, schedule number virtual machines to pe with low voltages and try not to scale pe with high voltages. Algorithm [14] is implemented with above assumptions. Power $\mathrm{p}$ could be reduced when supplied $\mathrm{v}$ is minimum. Operational scenario of algorithm is shown in Figure 2.

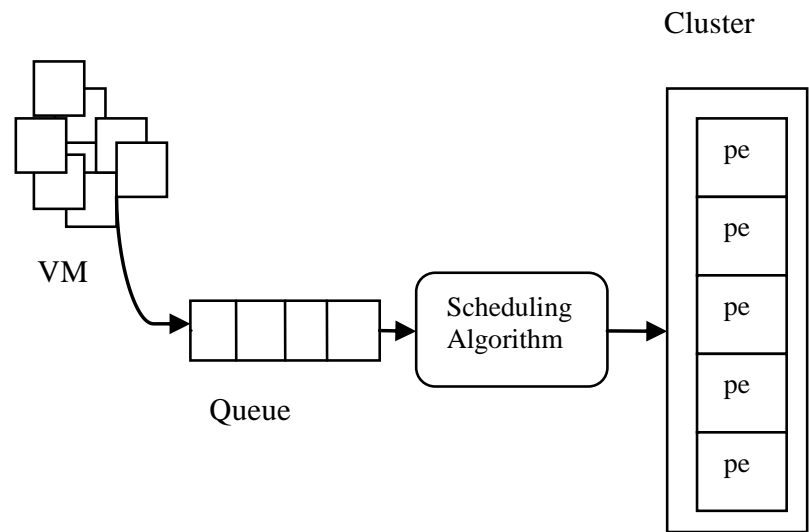

Fig 2. Operating Scenario of a DVFS-enabled cluster scheduling

\subsection{Energy-Aware Heuristic Algorithm}

Bo Li [20], [25], [26], [27] proposed a novel approach called EnaCloud on base of Energy saving in cloud platform. The energy-aware heuristic algorithm [20] is developed to get perfect solution for less energy consumption. Algorithm contains workload migration, virtual machine migration and resize of workload. Open node means active node or virtual machine in $\mathrm{ON}$ state. Close node means that virtual machine is shutdown or in CLOSE state. When workload arrives at that time do not start new virtual machine or close node and distribute that workload on already open nodes. If number of open nodes are minimum and fully distribution of total workload on minimum number of open nodes, maximum number of close node then power required less. So obviously $10 \%$ to $15 \%$ energy saved when workload complete totals.

Workload resizing includes workload inflation and workload deflation [20]. The migration has two goals i) minimize number of open nodes ii) minimize the times of migration, when workload arrives. The principle is inserting it into open node without opening close node. The algorithm based on heuristic rule that small workload are likely to inserted into the gaps so minimum number of open nodes will remain. Algorithm [20] idea is based on above description The complete process involves swap smaller workloads with larger workload and reinserted smaller ones, thus a lot of reinsertion operation required to reduce number reinsertions and lower complexity of algorithm divide workload size $(0,1]$ into 2M-2 subintervals according to partition method [21] each subinterval represents a level

$$
\begin{gathered}
\mathrm{L}_{0}=\left(\frac{(\mathrm{M}-1)}{\mathrm{M}}, 1\right] \\
\mathrm{L}_{1}=\left(\frac{(\mathrm{M}-2)}{(\mathrm{M}-1)}, \frac{\mathrm{M}-1}{\mathrm{M}}\right] \\
\mathrm{L}_{\mathrm{M}-1}=\left(\frac{1}{3}, \frac{1}{2}\right] \\
\mathrm{L}_{2 \mathrm{M}-4}=\left(\frac{1}{\mathrm{M}}, \frac{1}{\mathrm{M}-1}\right] \\
\mathrm{L}_{2 \mathrm{M}-3}=\left(0, \frac{1}{\mathrm{M}}\right]
\end{gathered}
$$

On base of these number of parameters, algorithm [20] work in three procedures Insert, Pop, Resize. In Insert procedure the size of new workload is calculated, using First-Fit method it is inserted in the gap of two workloads, Pop procedure workload migrates from source open node to destination open node. In resize is rather than a pop and Insert procedure, it can be transformed to a Pop and Resize procedure. Figure 3 shows procedure of algorithm.

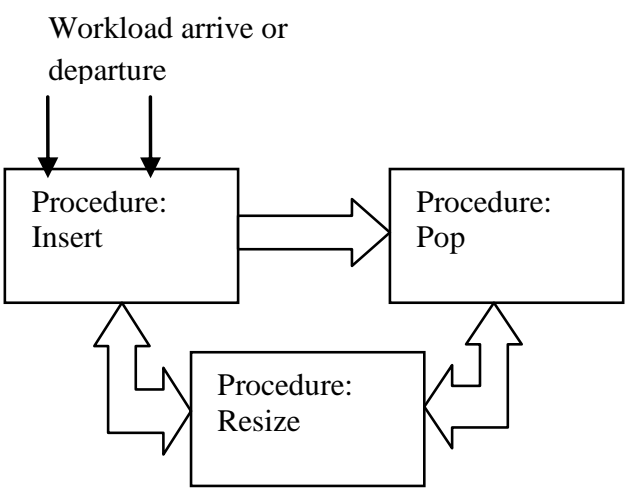

Fig 3. Procedure of Energy-Aware Heuristic Algorithm

\section{EXPERIMENTAL RESULTS AND COMPARISONS}

Currently, there is no detailed comparison among energy efficient algorithms which are recently proposed. For the purpose of comparison and to find better algorithm, we implement these methods according to published papers. To compare their performance, we apply energy parameter, evaluate each algorithm by their energy saving strategy, measured their energy to find compare which is better. We use 4 personal computers (HCL 3.06) each have LX INFINITI PRO BL 1205 CPU P4 524@3.06GH with 2GB memory connected to 10/100 Mbps switched Ethernet. Each host contain 4 virtual machines, host 1 acts as a scheduler, calculated Conserve Energy(E) in Joule(J), Response time(T) in second, voltage in $\mathrm{v}$, frequency in ( $\mathrm{f}$ ) in $\mathrm{GHz}$, Time (T) in second. Experimental results clearly demonstrate the effectiveness of hybrid energy efficient algorithm. We state that hybrid energy efficient algorithm is more energy efficient 
with maximum energy conserve i.e. $5997 \mathrm{~J}$, as compare to power aware scheduling algorithm and energy aware heuristic algorithm. Basically first method is based on parameter Response time $\mathrm{T}$, if $\mathrm{T}$ required is less then more energy conserve.

In Power-Aware scheduling algorithm, incoming virtual machine request are scheduled in such a way that they will require low voltage and frequency. If it is low then energy consumption reduces, In Energy-Aware heuristic scheduling algorithm it schedules virtual machine workload in such a way that it requires less number of virtual machines. If less number of machines is used then power is conserved. The results clearly exhibit that Hybrid Energy efficient algorithm more superior than Power-Aware scheduling algorithm and Energy-Aware heuristic algorithm. However energy aware heuristic algorithm is better than Power-Aware scheduling algorithm.

Table 1 . Experimental Results

\begin{tabular}{|c|c|c|}
\hline $\begin{array}{c}\text { Methods/ } \\
\text { Algorithm }\end{array}$ & $\begin{array}{c}\text { Energy } \\
\text { conserve E in J }\end{array}$ & $\begin{array}{c}\text { Response / } \\
\text { Total Time } \\
\text { T }\end{array}$ \\
\hline $\begin{array}{c}\text { Hybrid Energy } \\
\text { Efficient algorithm }\end{array}$ & 5997 & 4881 \\
\hline $\begin{array}{c}\text { Power- Aware } \\
\text { scheduling } \\
\text { algorithm }\end{array}$ & 5430 & 5511 \\
\hline $\begin{array}{c}\text { Energy-Aware } \\
\text { Heuristic Algorithm }\end{array}$ & 5869 & 5321 \\
\hline
\end{tabular}

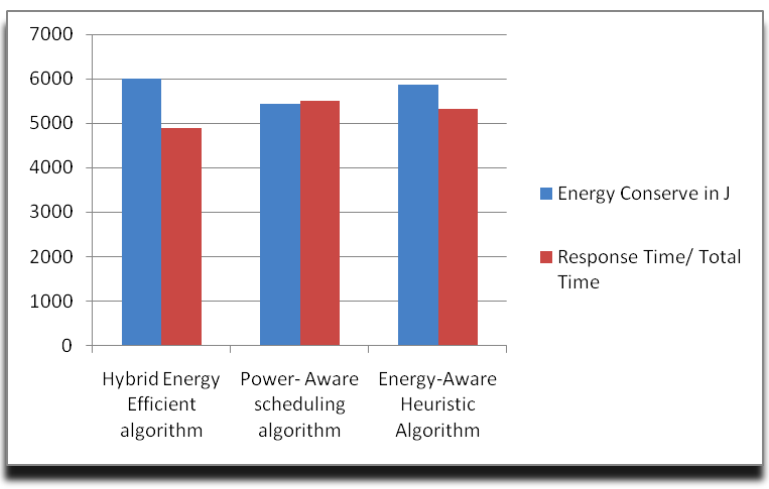

\section{Fig 4. Comparision of Conserved Energy In J}

\section{CONCLUSION}

From the experimental result illustrated above, it has been proved that Hybrid Energy efficient algorithm based on Response time is effective. Results show that it can not only reduce Response time ,conserve more energy but also achieves higher load balancing. In Energy-Aware Heuristic algorithm based on maximum workload distribution on minimum virtual machine and achieves $80 \%$ to $90 \%$ workload distribution, this algorithm is more powerful in dynamic applications.

\section{FUTURE SCOPE}

Future work includes developing scheduling scheme which is energy efficient, have less power consumption. It can fully distribute workload on less number of virtual machines using virtual machine migration, cloning of virtual machine, and workload application migration. This scheme shall be used effectively used in Infrastructure as a Service, Platform as a Service and Software as a service of cloud computing.

\section{REFERENCES}

[1] M. Sheikhalishahi, M.Devare, L. Grandinetti (2011), "A General purpose And Multi-level Scheduling Approach in Energy Efficient Algorithm", CLOSER Conference.

[2] Susane Albers (2010),"Energy efficient algorithms", Communication of ACM, vol.53 No.5, 86-96.

[3] D.Nurmi, R.Wolski, C.Grzgorczyk (2009), "The eculyptus open-source cloud-computing system", In Proc. Of CCGRID’09, 124-131. http://en.wikipedia,org/wiki/Round_robin_dchedilorg.

[5] Greedy on Wikipedia, http://en.wikipedia.org/wiki/Greedy_algorith.

[6] M. Devare, M .sheikhalishahi, L.Grandinetti (2010) ,' Prototype Implementation of Desktop cloud ", by IOS press, Netherland ,presented in Centraro International workshop on HPC, Grids and clouds .

[7] M. Devare , M. sheikhalishahi , L.Grandinetti (2009)," Virtualization: A Foundation for Cloud Computing ",International Journal of Networking and communication and Engineering .

[8] Jiandun Li, Junjie Peng, Wu Zhang (2011),"An Energyefficient Scheduling Approach Based on Private Clouds" , Journal of Information \& Computational Science ,volume 8, Number 4 , 716-724.

[9] Jiandun Li, Junjie Peng, Wu Zhang (2011), "A Scheduling Algorithm for Private Clouds", Journal of Convergence Information Technology, Volume 6, Number 7, 1-9.

[10] Saurabh Kumar Garg, Chee Shin Yeo, Arun Anandasivam, Rajkumar Buyya (2011) "Environmentconscious scheduling of HPC applications on distributed cloud-oriented data centers ", Journal of Parallel and Distributed Computing, vol.71, no.6, 732-749.

[11] Akshat Verma, Puneet Ahuja, Anindya Neogi (2008), "Power-aware dynamic placement of HPC applications", Proceedings of the 22nd International Conference on Supercomputing (ICS'08), Island of Kos, 175-184.

[12] Chuling Weng, Zhigang Wang, Minglu Li, and Xinda Lu (2009), "The Hybrid Scheduling Framework for Virtual Machine Systems ,"Proc. Conf. VEE09, 113-120.

[13] Gaurav Dhiman ,Giacomo Marchetti ,Tajana Rosing (2009),"vGreen: A System for Energy Efficient Computing in Virtualized Environments", In conference of ISLPED 2009 San Francisco,California ,USA,19-21 .

[14] Gregor von Laszewski ,LizheWang ,Andrew J. Younge ,Xi He (2009) ,"Power-Aware Scheduling of Virtual Machines in DVFS-enabled clusters" ,cluster 09 IEEE international on Cluster , 1-11. 
[15] W.Feng and K.Cameron(2007), " The Green500 List:Encouraging Sustainable Supercomputing “, IEEE Computer, 50-55.

[16] W. Feng and X.Feng(2009), "Green supercomputing comes of age,” IT Professional, vol 10 no 1 ,17-23.

[17] D. Bodas (2003), " Data Center Power Management and Benefits to Modular Computing “, In Intel Developer Forum Available

at http://www.intel.com/idf/us/spr2003/presentations/SO3U SMODS137.

[18] K. H. Kim, R.Buyya and J.kim (2007), "Power aware Scheduling of Bag-of-Tasks Applications with Deadline Constriants on DVFS-enabled Grids"in CCGRID , 541548.

[19] R. Ge, X. Feng and K. Cameron (2005), "Performanceconstrained distributed dvs scheduling for scientific application on power-aware clusters ",In Proceeding of the 2005 ACM/IEEE conference Supercomputing IEEE Computer Society Washington, DC, USA.

[20] Bo Li, Jianxin Li, Jinpeng Huai, Tianyu Wo, Qin Li,Liang Zhong (2009), "EnaCloud:An Enegy-saving Application Live Placement Approach for Cloud Computing Enviorments" ,In IEEE Intenational Conference on cloud Computing 200, 17-24.

[21] C. C. Lee and D. T. Lee (1985), "A simple on-line binpacking algorithm ", journal of the ACM ,32(3):562572.

[22] Jan Stoess, Christian Lang, Frank Bellosa (2007), "Energy management for hypervisor-based virtual machines ", in proceedings of the 2007 USENIX Annual Technical Conference (USENIX ‘07) pp.1-14 .

[23] Jeffery O. Kephart, Hoi Chan, Tajashri Das (2007), "Coordinating multiple automatic managers to achieve specified power-performance tradeoffs “ "In proceedings of the 4th International Conference on Automatic Computing (ICAC'07) ,22-33.
[24] Hien Nguyen Van, Frederic Dang Tan ,Jean-Marc Menaud (2010), "Performance and power management for cloud infrastructures ", In proceeding of IEEE 3rd International Conference on Cloud Computing ,329-336.

[25] C. Clark, K. Fraser, S.Hand, J.G.Hansen, E.Jule ,C. Limpach, I. Pratt and A. Warfield (2005), "Live migration of Virtual machines", Proceedings of 2nd Symposium on Network System Design and Implementation ,USENIX .

[26] C. C. Lee and D. T. Lee (1985) , "A simple on-linepacking algorithm ",Journal of the ACM ,32(3):562-572.

[27] Rajamani, K. Lefurgy ,C. (2003), “ on evaluating request-distribution schemes for energy saving in server cluster ", In proceeding of the 2003 IEEE International Symposium on Performance Analysis of Systems and software.

[28] V. Buge, Y.Kemp, M.Kunze, and G.Quest (2006), "Application of Virtulisation Techniques at a University Grid center", e-Science, 155-155.

[29] N. Fallenbeck, H. Pitch, M. Smith, and B. Freisleben (2006) , "Xen and the art of cluster scheduling", in First International Workshop on virtulization Technology in Distributed computing,4-4.

[30] H. N. Van, F. D Tran, J.M.Menaud (2010), "Performance and power management for cloud infrastructure ", In Proc. Of CLOUD 2010, 329-336.

[31] R. V. Patil, K. C. Jondhale (2010)," Edge based technique to estimate number of clusters in k-means color image segmentation", IEEE Internatinal conference on Computer Sciene and Information Technology, Chengdu , China, vol. 2, pp. 117-121

[32] P. S. Patil, S. R. Kolhe, R. V. Patil, P. M. Patil (2012), "Performance Evaluation in Iris Recognition and CBIR System based on phase congruency", International Journal of Computer Applications, 47(14), 13-18. 